\title{
Make the most of qualitative research
}

\author{
Nature Sustainability aims to give qualitative studies the recognition they deserve.
}

T $o$ address grand sustainability challenges, we need more than numbers alone. A lot of knowledge is uncovered through qualitative means only. Such methods can be more practicable to address urgent issues or where large samples are difficult to access. Crucially, often few people are pivotal to gain certain insight, so a large- $\mathrm{N}$ survey or experiment cannot replace a rich and nuanced interview or focus group discussion.

There are ways to enhance the potential of qualitative research to contribute to sustainability. The first step is to give qualitative evidence the scholarly and practical recognition it deserves. Doing so requires adequate understanding of its value to support practice, for example using the principles to balance evidence types proposed by Game and colleagues in a Comment in this journal.

Second, we need to share qualitative data more - for example, in public repositories, for it to support and inspire further

knowledge. However, this sharing is rare relative to that of quantitative data. In this issue, Alexander and colleagues discuss in a Perspective why that's the case and what each actor involved in the generation and diffusion of science can do to enhance qualitative data sharing.

Third, alongside quantitative publications we need qualitative papers, and they can share the same format. In this issue, we present two such examples: combining expert interviews and document analysis, Miller and colleagues identify key barriers to implement prescribed burns - a measure that can reduce wildfire impact; and, using a mixedmethods approach, Rosenberg and colleagues find evidence about the link between gender inequality and energy access in India.

A number of features in these two papers can help and encourage researchers in preparing their qualitative work for publication in strong interdisciplinary journals. Qualitative papers can be concise and their terminology accessible - and this will certainly help the study reach more readers. Further, qualitative research does have methods that can be explained in a dedicated section, much like quantitative studies. Readers and peer reviewers need to know how this new knowledge was produced, for example, how interviewees were selected, why they were questioned about certain themes, what set of documents were analysed, or how the data were coded. Finally, presenting creatively qualitative evidence - such as direct quotations from research subjects - can make a real difference when writing concisely and for a broad audience. Crafting a cohesive and engaging narrative is remarkably hard if it intertwines with numerous direct quotations (much like prose filled with point estimates and $P$ values). Moving those quotations to be in display items - and referring to them throughout the text - can enhance communication.

Since its launch, Nature Sustainability has been open to all research that can contribute to sustainability in meaningful ways, regardless of methodological approach - and here we reinforce this commitment.

Published online: 14 February 2020 https://doi.org/10.1038/s41893-020-0482-0

\section{Sustainable water solutions}

\author{
Engineering approaches to wastewater treatment must aim for more than improved efficiency.
}

W ater is essential to any form of life on Earth - we need enough of it and of the right quality. This is so much at the core of sustainability that we have written about it in previous editorials (Nat. Sustain. 1, 151-152 (2018) and Nat. Sustain. 1, 447 (2018)). Research has long documented the severe impacts that human activities have on both water availability and quality, including in Nature Sustainability more recently. We also know that significantly reducing those impacts is far from easy, especially against prospects of a growing population globally — our diets are based on water, a large portion of energy activities need water in various ways, most manufacturing and consumption activities require some form of water use and, all such activities ultimately produce some kind of wastewater. And it is precisely on wastewater - and how to treat it sustainably - that we want to focus our readers' attention with this editorial. Given the different forms of water contamination, society needs to identify and implement different solutions. In this issue, we highlight three cases: treatment of wastewater from the oil industry in an Article by Park and colleagues, treatment of desalination brine in an Article by Prasher and collaborators and finally, treatment of water contaminated by glyphosate use in an Article by Halik and co-authors. Park and colleagues show how reusable surfaceengineered sponges offer a sustainable solution to efficiently remove crude oil microdroplets from wastewater. Prasher and collaborators demonstrate a scalable surface-heating approach to improve solar evaporation in brine-disposal ponds, usually requiring large surface areas to passively evaporate water for the removal of brine - the solution significantly reduces the need of land to treat wastewater. Halik and co-authors present a method for the efficient remediation of the herbicide glyphosate from water by means of inexpensive, recyclable magnetite nanoparticles. All three studies have some common sustainability features. Of course, the three different solutions discussed have the potential to increase the efficiency of water treatment with respect to commonly used methods. But beyond the improved efficiency, each approach presents one or more additional advantages. Reusability, recyclability, scalability, low cost or reduced need of primary inputs (for example, land), are critical sustainability gains if we embrace an integrated view of what sustainable solutions must be. It is therefore the combination of improved efficiency with any of those gains that makes for socially desirable solutions to the water challenges of our time. We at Nature Sustainability call for more research focusing on such holistic solutions.

Published online: 14 February 2020 https://doi.org/10.1038/s41893-020-0481-1 